\title{
Clinical features and outcomes of patients with hepatocellular carcinoma complicated with bile duct invasion
}

\author{
Jihyun $\mathrm{An}^{1,2^{*}}$, Kwang Sun Lee ${ }^{1,2^{*}}$, Kang Mo Kim ${ }^{1,2}$, Do Hyun Park ${ }^{2}$, Sang Soo Lee ${ }^{2}$, Danbi Lee ${ }^{1,2}$, Ju Hyun Shim ${ }^{1,2}$, \\ Young-Suk Lim ${ }^{1,2}$, Han Chu Lee ${ }^{1,2}$, Young-Hwa Chung ${ }^{1,2}$, and Yung Sang Lee ${ }^{1,2}$ \\ ${ }^{1}$ Department of Internal Medicine, ${ }^{2}$ Asan Liver Center, Asan Medical Center, University of Ulsan College of Medicine, Seoul, Korea
}

Background/Aims: Little is known about the treatment or outcomes of hepatocellular carcinoma (HCC) complicated with bile duct invasion.

Methods: A total of 247 consecutive HCC patients with bile duct invasion at initial diagnosis were retrospectively included.

Results: The majority of patients had Barcelona Clinic Liver Cancer (BCLC) stage C HCC (66.8\%). Portal vein tumor thrombosis was present in 166 (67.2\%) patients. Median survival was 4.1 months. Various modalities of treatment were initially employed including surgical resection (10.9\%), repeated transarterial chemoembolization (TACE) (42.5\%), and conservative management (42.9\%). Among the patients with obstructive jaundice $(n=88)$, successful biliary drainage was associated with better overall survival rate. Among the patients with $B C L C$ stage $C$, overall survival differed depending on the initial treatment for HCC; surgical resection, TACE, systemic chemotherapy, and conservative management showed overall survival rates of $11.5,6.0,2.4$, and 1.6 months, respectively. After adjusting for confounders, surgical resection and repeated TACE were significant prognostic factors for $\mathrm{HCC}$ patients with bile duct invasion (hazard ratios 0.47 and 0.39 , Ps $<0.001$, respectively).

Conclusions: The survival of HCC patients with bile duct invasion at initial diagnosis is generally poor. However, aggressive treatments for HCC such as resection or biliary drainage may be beneficial therapeutic options for patients with preserved liver function. (Clin Mol Hepatol 2017;23:160-169)

Keywords: Hepatocellular carcinoma; Bile duct invasion; Treatment; Prognosis; Obstructive jaundice

\section{INTRODUCTION}

Hepatocellular carcinoma (HCC) is the sixth most common cancer worldwide and the third most frequent cause of cancer death. ${ }^{1}$
Vascular invasion, especially invasion of the portal vein, has a well-documented negative impact on survival in patients with HCC. ${ }^{2,3}$ However, only a few sporadic reports have been published on HCC patients with bile duct invasion. Its incidence is $1.2 \%$ to

\footnotetext{
Abbreviations:

AASLD, American Association for the Study of Liver Disease; AJCC, American Joint Committee on Cancer; ANOVA, one-way analysis of variance; BCLC, Barcelona Clinic Liver Cancer; CT, computed tomography; EASL, European Association for the Study of the Liver; HCC, hepatocellular carcinoma; MRI, magnetic resonance imaging; RT, radiation treatment; TACE, transarterial chemoembolization; TACL, transarterial chemolipiodolization

* Jihyun An and Kwang Sun Lee contributed equally to this work as cofirst authors.
}

\section{Corresponding author: Kang Mo Kim}

Department of Internal Medicine, Asan Liver Center, Asan Medical Center, University of Ulsan College of Medicine, 88 Olympic-ro 43-gil, Songpagu, Seoul 05505, Korea

Tel: +82-2-3010-3190; Fax: +82-2-485-5782

E-mail:kimkm70@amc.seoul.kr

http://orcid.org/0000-0002-3180-3178 
$9.0 \%$ according to the literature, mainly based on operative findings and autopsy cases. ${ }^{4-8}$

HCC patients with bile duct invasion are thought to have a poorer prognosis than those without bile duct invasion. ${ }^{8-10}$ Because HCC with bile duct invasion is often accompanied by obstructive jaundice, it is difficult to assess hepatic functional reserve before deciding on treatment. ${ }^{9,11}$ Cholangitis and hemobilia, which often develop in association with bile duct invasion, are also immediate threats to survival. In addition, HCC involvement of the bile duct could theoretically be accompanied by portal vein thrombosis, which is one of the worst prognostic factors for HCC patients. ${ }^{12}$

However, there is a paucity of data on the clinical characteristics, treatment options, and outcomes of HCC patients with bile duct invasion. A few previous studies only included patients receiving particular treatments, such as surgical resection, or endoscopic or percutaneous drainage. ${ }^{11,13-15}$ Even the American Joint Committee on Cancer (AJCC) and the Barcelona Clinic Liver Cancer (BCLC) classifications, which are most widely as staging systems for HCC, do not include the presence of bile duct invasion as a variable for staging. ${ }^{3,16}$

Hence, in this retrospective cohort study, we analyzed the clinical characteristics and outcomes of consecutive patients with HCC and bile duct invasion at the time of initial diagnosis. We also sought to identify prognostic factors for survival in these patients.

\section{MATERIALS AND METHODS}

\section{Study population}

This retrospective cohort study was based on 6,663 consecutive patients diagnosed with HCC at our institution from January 2006 and December 2010 (Fig. 1). Bile duct dilatation adjacent to the primary tumor on initial four-phase computed tomography (CT) was observed in 985 of these patients. Bile duct dilatation was defined as a common duct diameter greater than $6 \mathrm{~mm}$ and an intrahepatic duct diameter greater than $3 \mathrm{~mm}$ as considered the upper limit of normal diameters of each bile duct in previous studies. ${ }^{17,18}$ Patients previously treated for $\mathrm{HCC}$ at other hospitals $(\mathrm{n}=615)$ were excluded, as were subjects with bile duct dilatation not caused by HCC, such as bile duct stone, post cholecystectomy state, or Clonorchis sinensis infection ( $n=123$ ). Finally, 247 patients with suspected bile duct invasion by $\mathrm{HCC}$ on $\mathrm{CT}$ at initial diagnosis were included in the study.

The diagnosis of HCC was based on the American Association for the Study of Liver Disease (AASLD) and European Association for the Study of the Liver (EASL) guidelines with either pathological or radiological findings. ${ }^{19,20}$ Bile duct invasion by HCC was diagnosed based on $\mathrm{CT}$ and/or magnetic resonance imaging (MRI), and classified according to its location such as segmental, unilateral first-order hepatic duct, or bilateral involvement. The presence and extent of portal vein tumor thrombus were assessed by dynamic CT: enhancement with neo-vascularity in the portal vein on the arterial phase, along with intraluminal low-attenuated filling defect adjacent to the primary tumor on the portal-delayed phase. The study was undertaken with the approval of the local research ethics committee, and in accordance with the Declaration of Helsinki (1989) of the World Medical Association.

\section{Treatment modalities}

Study subjects received either endoscopic or percutaneous bili-

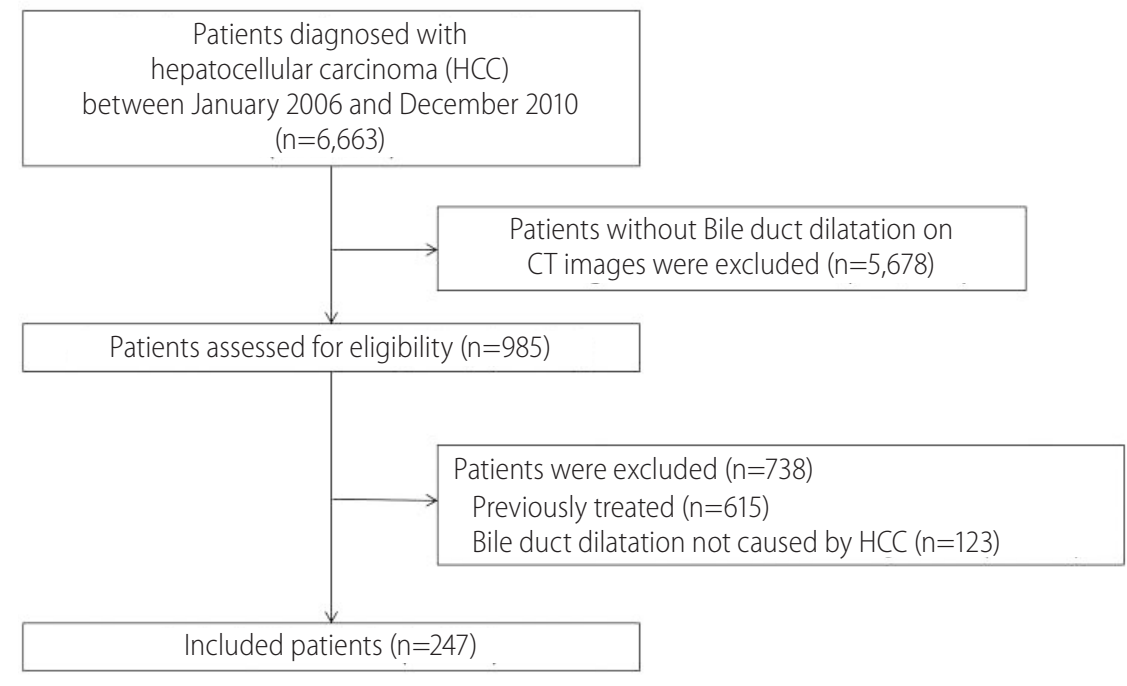

Figure 1. Patient flow diagram. HCC, hepatocellular carcinoma; CT, computed tomography. 
ary drainage depending on the clinicians' judgment. Drainage was conducted in the standard manner. The function of bile duct drainage was evaluated within one month of HCC diagnosis in patients with obstructive jaundice (serum total bilirubin concentration $\geq 3 \mathrm{mg} / \mathrm{dL}$ ). Successful drainage was defined as a decrease in the total bilirubin concentration of more than $50 \%$ of the preprocedural value, or a decrease to $<3 \mathrm{mg} / \mathrm{dL}$ within 4 weeks, and no cholangitis. ${ }^{11,21-24}$

Treatment for HCC of each patient was mainly decided in a hierarchical manner according to its effect in lengthening life. Specifically, surgical resection was carried out based on anatomical segment of the liver, if technically feasible and preserved liver function. Transarterial chemoembolization (TACE) was considered the main treatment for patients whom curative strategies such as resection, ablation or liver transplantation were contraindicated or ineligible at the time of study enrolment. Because of the risk of hepatic failure after TACE, transarterial chemolipiodolization (TACL) without gelatin sponge particle embolization (Gelfoam; Upjohn, Kalamazoo, MI, USA) was performed in case of some patients with main portal vein invasion or Child-Pugh class B liver function. TACE was repeated every $6-8$ weeks, if residual viable tumor tissue was evident at sequential four-phase CT or MRI without deterioration of hepatic function.

Radiation treatment (RT), in addition, was given to individuals with gross portal vein invasion after one TACE/TACL session. ${ }^{25}$ The dose and method of RT was the same as that in a previous report. ${ }^{25}$ Sorafenib was combined with TACE/TACL as the initial treatment in some patients, depending on the physicians' decision and patients' informed consent. Systemic chemotherapy such as 5-fluorouracil, cisplatin, doxorubicin, gemcitabine, oxaliplatin or sorafenib was given to patients in the Child-Pugh class A-B liver function group and those refusing TACE. Patients with poor liver function (Child-Pugh $C$ at presentation) or those denied treatment, received only conservative management.

\section{Data collection}

The clinical, laboratory, and radiological data for all patients were reviewed retrospectively. Initial laboratory evaluations included assessments of underlying liver disease by serum hepatitis B surface antigen and hepatitis $C$ antibody, and of parameters of liver function such as albumin, bilirubin and prothrombin time. Radiological evaluation included size of tumor(s) and numbers of tumors in liver, lymph nodes or distant metastasis, and sites of

Table 1. Baseline characteristics of patients with hepatocellular carcinoma complicated with bile duct invasion at the time of initial diagnosis ( $\mathrm{N}=247$ )

\begin{tabular}{lc}
\hline Variable & Value \\
\hline Age (years) & $56(49-64)$ \\
Sex, male & $206(83.4)$ \\
\hline Etiology, HBV/HCV/alcohol/others & $176 / 18 / 14 / 39(71.2 / 7.3 / 5.7 / 15.8)$ \\
\hline Child-Pugh class, A/B/C & $115 / 111 / 21(46.6 / 44.9 / 8.5)$ \\
\hline Serum total bilirubin & $1.6(1.0-5.3)$ \\
\hline$<3.0 / \geq 3.0$ mg/dL & $159 / 88(64.4 / 35.6)$ \\
\hline Serum alpha-fetoprotein (ng/mL) & $957.5(49.4-25825.0)$ \\
\hline Tumor number, single/multiple & $94 / 153(38.1 / 61.9)$ \\
\hline Tumor size (cm) & $8.0(5.2-11.0)$ \\
\hline PVTT, none/unilateral/main or bilateral & $81 / 86 / 80(32.8 / 32.4 / 34.8)$ \\
Level of bile duct involvement & $74(30.0)$ \\
\hline Segmental bile duct & $82(33.2)$ \\
\hline Unilateral 1st brach duct & $91(36.8)$ \\
\hline Bilateral or common bile duct & $53(21.4)$ \\
\hline Extra-hepatic metastasis & $25 / 20 / 5 / 3(10.1 / 8.1 / 2.0 / 1.2)$ \\
\hline Lung / lymph node / bone / adrenal gland & $33 / 29 / 165 / 20(13.4 / 11.7 / 66.8 / 8.1)$ \\
\hline BCLC stage, A/B/C/D &
\end{tabular}

All values are expressed as median (interquartile range) or number (\%).

HBV, hepatitis B virus; HCV, hepatitis C virus; PVTT, portal vein tumor thrombus; BCLC, Barcelona Clinic Liver Cancer. 
portal vein and bile duct invasion on initial HCC diagnosed by CT. Information on initial and subsequent HCC treatments was also collected from the medical records.

\section{Statistical analysis}

The primary end-point was survival from the date of diagnosis. Survival time was defined as time from the date of imaging showing bile duct invasion by HCC to the date of death or the last follow-up. Survival curves for all patients, and subgroups sorted by $\mathrm{BCLC}$ stage or treatment modality, were estimated by the KaplanMeier method, and compared by log-rank tests. Baseline characteristics and laboratory data were presented as median and range for continuous variables, and as number of subjects (percentages) for categorical variables, unless otherwise stated. Student's $t$-test or one-way analysis of variance (ANOVA) was used to analyze differences between groups, and Fisher's exact test for comparisons between categorical variables. To identify factors affecting patient survival, multivariate analysis using the Cox regression model was performed. A $P$-value $<0.05$ was considered significant for all statistical tests. Statistical analyses were performed with SPSS version 21.0 software (SPSS Inc., Chicago, IL, USA).

\section{RESULTS}

\section{Patient characteristics}

The baseline characteristics of the included patients are summarized in Table 1. Of the 247 patients, 206 (83.4\%) were male, and median age was 56 years (range, 30-84 years). The major cause of underlying liver disease was hepatitis B virus infection (71.3\%). The classification of HCC according to BCLC stage was as follows: 33 (13.4\%) cases of BCLC A; $29(11.7 \%)$ cases of BCLC
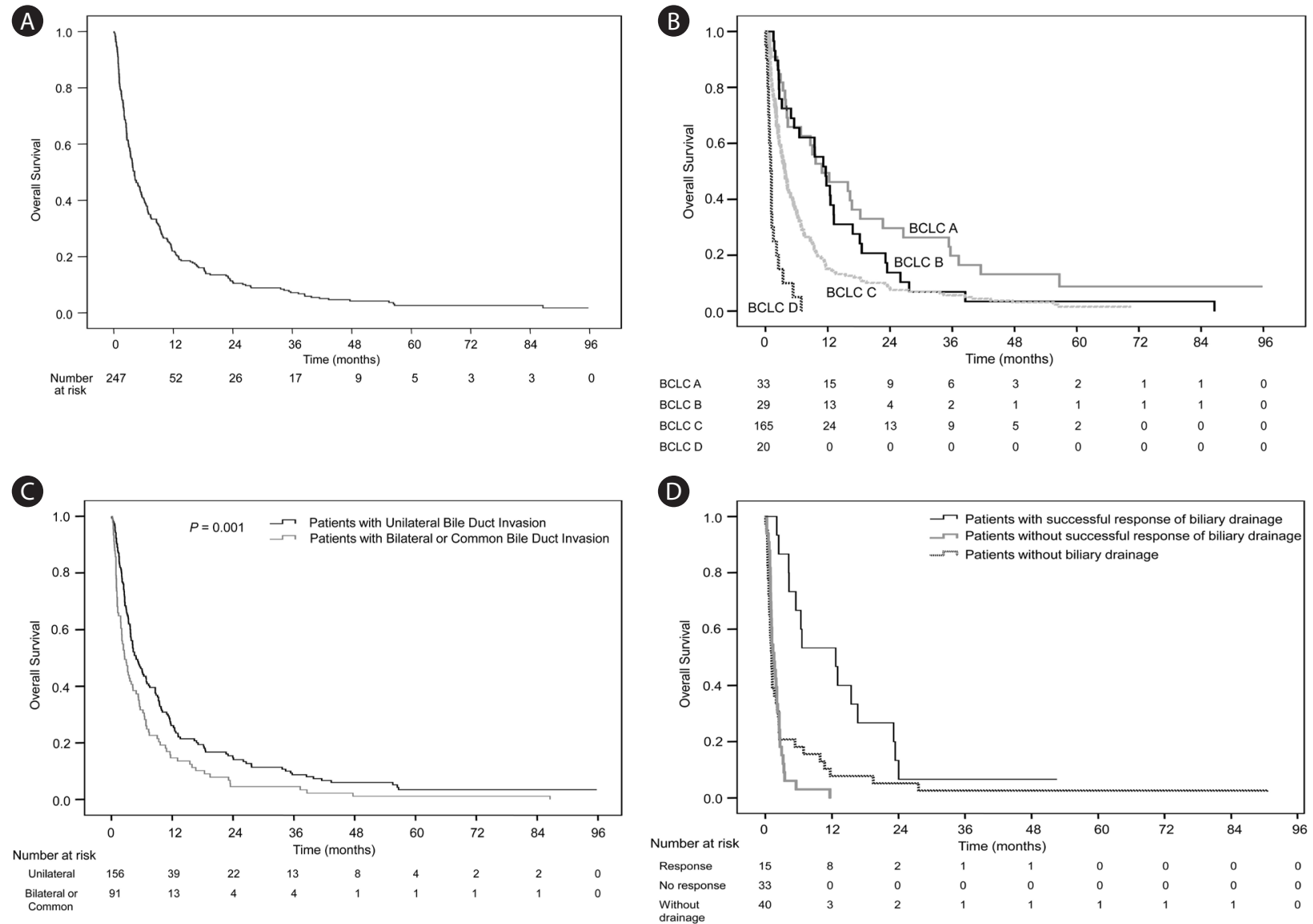

Figure 2. Overall survival of patients with hepatocellular carcinoma complicated with bile duct invasion at the time of diagnosis. (A) Overall survival $(n=247)$. (B) Overall survival according to Barcelona Clinic Liver Cancer (BCLC) stage $(n=247)$. (C) Overall survival according to the level of bile duct invasion ( $n=247$ ). (D) Overall survival of 88 patients with obstructive jaundice, as a function of biliary drainage. 
B; $165(66.8 \%)$ cases of BCLC C; and $20(8.1 \%)$ of BCLC D. Most patients ( $n=226,91.5 \%$ ) had Child-Pugh A or B liver function. Portal vein tumor thrombosis was evident in 166 (67.2\%) patients. CT images pointed to invasion of bilateral or common bile duct, 1st branch of unilateral duct, or segmental branch of duct in $91(36.8 \%), 82(33.2 \%)$ and $74(30.0 \%)$ patients, respectively. Fifty three $(21.5 \%)$ patients had extra-hepatic metastasis of the $\mathrm{HCC}$ at initial diagnosis.

\section{Survival analysis}

Of the 247 patients, 236 (95.5\%) died during follow-up. The median survival period was 4.1 months, and 1- and 2-year survival rates were $21.9 \%$ and $11.0 \%$, respectively (Fig. $2 \mathrm{~A}$ ). The major causes of death were cancer progression $(n=185,74.9 \%)$, sepsis $(n=20,8.1 \%)$, and gastrointestinal bleeding $(n=8,3.2 \%)$.

When we analyzed the survival of HCC patients with bile duct invasion according to $B C L C$ stage, the median survival periods of the
BCLC $A, B, C$ and $D$ groups were $10.8,11.6,3.7$, and 1.0 months, respectively, as shown in Figure $2 B(P=0.190$ for $B C L C A$ vs, $B$; $P<0.001$ for $B C L C$ stage $A$ vs. $B C L C$ stage $C ; P<0.001$ for $B C L C A$ vs. $B C L C$ stage $D ; P=0.013$ for $B C L C$ stage $B$ vs. $B C L C C ; P<0.001$ for $B C L C$ $B$ vs. BCLC $D$; and $P=0.001$ for $B C L C$ C vs. BCLC D).

The fraction of patients with portal vein invasion had shorter survival periods than those without portal vein invasion (median survival periods, 3.3 vs. 9.6 months, $P<0.001$ ). There was also a substantial difference in survival depending on the extent of bile duct invasion (unilateral vs. bilateral or common, with median periods of 4.9 and 2.6 months, respectively, $P=0.001$; Fig. 2C).

\section{Biliary drainage in HCC patients with bile duct invasion}

Obstructive jaundice defined as serum bilirubin $>3.0 \mathrm{mg} / \mathrm{dL}$ was observed in 88 (35.6\%) patients at initial diagnosis. Of these, 48 (54.5\%) were treated by biliary drainage within one month of diagnosis. Biliary drainage was not performed in the other patients

Table 2. Characteristics of patients according to initial treatment modality $(\mathrm{N}=247)$

\begin{tabular}{|c|c|c|c|c|c|}
\hline Variable & $\begin{array}{l}\text { Resection } \\
(n=27)\end{array}$ & $\begin{array}{c}\text { rTACE } \\
(n=105)\end{array}$ & $\begin{array}{c}\text { CTx } \\
(n=9)\end{array}$ & $\begin{array}{c}\text { CM } \\
(n=106)\end{array}$ & $P$-value \\
\hline Age (years) & $56(50-63)$ & $56(49-63)$ & $52(40-61)$ & $56(48-69)$ & 0.369 \\
\hline Sex, male & $19(70.4)$ & $90(85.7)$ & $9(100.0)$ & $88(83.0)$ & 0.137 \\
\hline Etiology, HBV & $18(66.7)$ & $74(70.5)$ & $8(88.9)$ & $76(71.7)$ & 0.640 \\
\hline Child-Pugh, A & $23(85.2)$ & $70(66.7)$ & $4(44.4)$ & $18(17.0)$ & $<0.001$ \\
\hline Total bilirubin (mg/dL) & $1.0(0.7-1.4)$ & $1.3(0.9-1.8)$ & $1.7(0.8-3.5)$ & $4.9(1.5-13.5)$ & $<0.001$ \\
\hline Total bilirubin $\geq 3.0 \mathrm{mg} / \mathrm{dL}$ & $3(11.1)$ & $18(17.1)$ & $2(22.2)$ & $65(61.3)$ & $<0.001$ \\
\hline AFP (ng/mL) & $\begin{array}{c}107.0 \\
(17.5-7,630.0)\end{array}$ & $\begin{array}{c}1,000.0 \\
(28.4-28,250.0)\end{array}$ & $\begin{array}{c}4,220.0 \\
(95.7-151,000.0)\end{array}$ & $\begin{array}{c}1,500.0 \\
(121.5-27,900.0)\end{array}$ & 0.560 \\
\hline Single tumor & $21(77.8)$ & $41(39.0)$ & $2(22.2)$ & $30(28.3)$ & $<0.001$ \\
\hline Tumor size $(\mathrm{cm})$ & $5.0(3.5-10.0)$ & $8.0(5.4-11.0)$ & $11.0(5.4-12.9)$ & $8.0(5.9-11.8)$ & 0.040 \\
\hline PVTT & & & & & $<0.001$ \\
\hline None & $18(66.7)$ & $37(35.2)$ & $1(11.1)$ & 25 (23.6) & \\
\hline Unilateral & $8(29.6)$ & $45(42.9)$ & $3(33.3)$ & $30(28.3)$ & \\
\hline Main or bilateral & $1(3.7)$ & $23(21.9)$ & $5(55.6)$ & $51(48.1)$ & \\
\hline Bile duct & & & & & $<0.001$ \\
\hline Common or both & $4(14.8)$ & $30(28.6)$ & $0(0.0)$ & $57(53.8)$ & \\
\hline Extra-hepatic metastasis & $0(0.0)$ & $23(21.9)$ & $6(66.7)$ & $24(22.6)$ & $<0.001$ \\
\hline $\mathrm{BCLC}$ stage & & & & & $<0.001$ \\
\hline$A / B / C / D$ & $\begin{array}{c}15 / 2 / 10 /- \\
(55.6 / 7.4 / 37.0 /-)\end{array}$ & $\begin{array}{c}11 / 16 / 77 / 1 \\
(10.5 / 15.2 / 73.3 / 1.0)\end{array}$ & $\begin{array}{c}-/-/ 8 / 1 \\
(-/-/ 88.9 / 11.1)\end{array}$ & $\begin{array}{c}7 / 11 / 70 / 18 \\
(6.6 / 10.4 / 66.0 / 17.0)\end{array}$ & \\
\hline
\end{tabular}

All values are expressed as median (range) or number (\%).

rTACE, repeated transarterial chemoembolization-containing regimen; CTx, systemic chemotherapy; CM, conservative management; HBV, hepatitis B Virus; AFP, alpha-fetoprotein; PVTT, portal vein tumor thrombus; BCLC, Barcelona Clinic Liver Cancer. 
with obstructive jaundice ( $n=40$ [45.5\%]) because hepatic insufficiency progressed too rapidly and the patients' conditions deteriorated, with hepatic encephalopathy and ascites. Internal drainage, external drainage and both forms of drainage were carried out in 18 (20.5\%), $17(19.3 \%)$ and $13(14.8 \%)$ patients, respectively, depending on the clinical context. Decreased serum bilirubin level was achieved in $15(31.3 \%)$ of these patients. The overall survival of these patients was better than that of the patients who did not have successful biliary drainage or who did not undergo the procedure (12.9 vs. 1.53 months, and 12.9 vs. 1.13 months. $P<0.001$, $P=0.003$, respectively; Fig. 2D). Most of patients for whom biliary drainage was unsuccessful received only conservative management.

\section{Modalities of treatment of HCC patients with bile duct invasion}

The characteristics of HCC patients with bile duct invasion according to initial treatment were shown in Table 2. Twenty-seven patients (10.9\%) underwent surgical resection with curative intent. Hepatocellular carcinoma was pathologically identified in the all the resected specimen. Of the patients, 4 (14.8\%) underwent biliary drainage before curative hepatectomy. With regard to tumor factors, capsular infiltration, microscopic vascular invasion, and satellite nodules were recorded histologically in 6 (22.2\%), 13 (48.1\%), and $8(29.6 \%)$ patients, respectively. A majority of the patients $(77.8 \%)$ had Edmondson grade III or IV tumors. One showed poorly differentiated type of HCC. The subsequent treatments of the 17 recurred patients were done as follows: TACE alone $(n=8)$, TACE plus radiotherapy $(n=2)$, TACE plus chemotherapy $(n=1)$, chemotherapy alone $(n=5)$, and photodynamic therapy of the bile duct $(n=1)$. Two patients without recurrence of HCC received TACE or systemic therapy as adjuvant treatments; one had a positive resection margin and the other had severe preoperative portal vein thrombosis. During the follow-up, seven out of eight patients without recurrence died due to complication of cirrhosis $(n=4)$, and other causes including tuberculosis $(n=3)$.

Repeated TACE as initial treatment was performed in 105 $(42.5 \%)$ of the patients. Of these, 46 were treated with RT confined to the gross vascular invasion, and 11 received sorafenib. Conventional systemic chemotherapy consisting of 5-fluorouracil, cisplatin, doxorubicin, gemcitabine, or oxaliplatin alone, was pro-

Table 3. Baseline characteristics of patients with $B C L C$ stage $C$ according to main treatment modality $(N=165)$

\begin{tabular}{|c|c|c|c|c|c|c|}
\hline Variable & $\begin{array}{c}\text { Total } \\
(n=165)\end{array}$ & $\begin{array}{c}S R \\
(n=10)\end{array}$ & $\begin{array}{l}\text { rTACE } \\
(n=77)\end{array}$ & $\begin{array}{c}\text { CTx } \\
(n=8)\end{array}$ & $\underset{(n=70)}{C M}$ & $P$-value \\
\hline Age (years) & $55(30-82)$ & $52(42-63)$ & $56(30-82)$ & $49(35-68)$ & $56(35-78)$ & 0.384 \\
\hline Sex, male & 138 (83.6) & $5(50.0)$ & 65 (84.4) & $8(100.0)$ & $60(85.7)$ & 0.018 \\
\hline Etiology, HBV & $123(74.5)$ & $8(80.0)$ & $56(72.7)$ & $7(87.5)$ & $52(74.3)$ & 0.801 \\
\hline Child-Pugh, A & $73(44.2)$ & $9(90.0)$ & $49(63.6)$ & $4(50.0)$ & $11(15.7)$ & $<0.001$ \\
\hline Total bilirubin $\geq 3.0 \mathrm{mg} / \mathrm{dL}$ & $54(32.7)$ & $1(10.0)$ & $13(16.9)$ & $1(12.5)$ & $39(55.7)$ & $<0.001$ \\
\hline Single tumor & 55 (33.3) & $6(60.0)$ & $28(36.4)$ & $2(25.0)$ & $19(27.1)$ & 0.174 \\
\hline Tumor size (cm) & $8.5(1.2-22)$ & $7.2(1.2-15)$ & $8.0(2.7-22)$ & $9.8(3.8-13)$ & $8.7(2.0-22)$ & 0.442 \\
\hline PVTT & & & & & & 0.002 \\
\hline None & $16(9.7)$ & $1(10.0)$ & $10(13.0)$ & $1(12.5)$ & $4(5.7)$ & \\
\hline Unilateral & $78(47.3)$ & $8(80.0)$ & 44 (57.1) & $3(37.5)$ & $23(32.9)$ & \\
\hline Main or bilateral & $71(43.0)$ & $1(10.0)$ & $23(29.9)$ & $4(50.0)$ & $43(61.4)$ & \\
\hline \multicolumn{7}{|l|}{ Bile duct } \\
\hline Common or both & $60(36.4)$ & $1(10.0)$ & $20(26.0)$ & $0(0.0)$ & $39(55.7)$ & $<0.001$ \\
\hline Extra-hepatic metastasis & & & & & & 0.272 \\
\hline Lung & $24(14.5)$ & $0(0.0)$ & $9(11.7)$ & $3(37.5)$ & $12(17.1)$ & \\
\hline Lymph node & $19(11.5)$ & $0(0.0)$ & $12(15.6)$ & $1(12.5)$ & $6(8.6)$ & \\
\hline Bone & $5(3.0)$ & $0(0.0)$ & $1(1.3)$ & $1(12.5)$ & $3(4.3)$ & \\
\hline
\end{tabular}

Values are represented as median (range) or $\mathrm{n}(\%)$.

BCLC, Barcelona Clinic Liver Cancer; SR, surgical resection-containing regimen; rTACE, repeated transarterial chemoembolization-containing regimen; CTx, systemic chemotherapy; CM, conservative management; HBV, hepatitis B virus; PVTT, portal vein tumor thrombus. 
vided for 9 (3.7\%) patients who refused TACE. One hundred six patients $(42.9 \%)$ received only conservative management because they had advanced HCC such as more than BCLC C stage or ChildPugh class C liver function, or they were elderly or refused treatment. In terms of bile duct complication after treatment, biloma was occurred in five patients. All of these patients underwent TACE as a treatment of HCC.

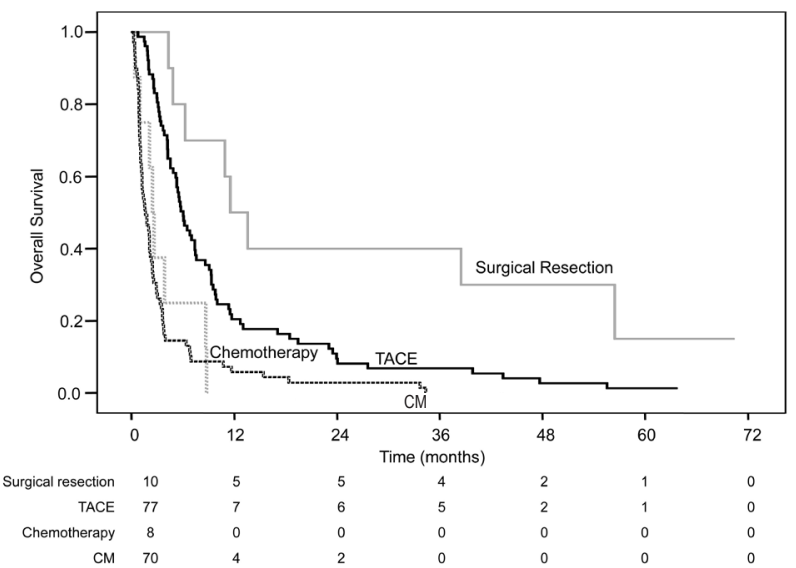

Figure 3. Overall survival of 165 patients with HCC BCLC stage $C$ and bile duct invasion as a function of treatment modality. HCC, hepatocellular carcinoma; BCLC, Barcelona Clinic Liver Cancer; TACE, transarterial chemoembolization-containing regimen; $\mathrm{CM}$, conservative management.

\section{Subgroup analysis of BCLC stage C patients}

The baseline characteristics of the BCLC stage $C$ patients $(n=165)$ by initial treatment of HCC are shown in Table 3. The most common treatment modality was repeated TACE, which was performed in $77(46.7 \%)$ patients. Ten (6.1\%) of the BCLC stage C patients received surgical resection. The characteristics of the patients receiving the different treatments were significantly different, especially in terms of Child-Pugh class, presence of obstructive jaundice, tumor number, and presence of portal vein invasion. Among the patients with $B C L C$ stage $C$, overall survival was significantly different according to the initial treatment modality, as presented in Figure 3. The median survival of patients who underwent surgical resection, TACE, systemic chemotherapy or conservative management were $11.5,6.0,2.4$, or 1.6 months, respectively ( $P=0.009$ for surgical resection vs. TACE; $P<0.001$ for surgical resection vs. systemic chemotherapy; $P<0.001$ for surgical resection vs. conservative management; $P=0.005$ for TACE vs. systemic chemotherapy; $P<0.001$ for TACE vs. conservative management; and $P=0.497$ for systemic chemotherapy vs. conservative management).

\section{Factors affecting the survival of HCC patients with bile duct invasion}

As shown in Table 4, in a univariate analysis to determine the

Table 4. Multivariate analysis of variables affecting the survival of HCC patients with bile duct invasion at the time of initial diagnosis ( $\mathrm{N}=247)$

\begin{tabular}{|c|c|c|c|c|}
\hline \multirow{2}{*}{ Variables } & \multicolumn{2}{|c|}{ Univariate analysis } & \multicolumn{2}{|c|}{ Multivariate analysis } \\
\hline & HR $(95 \% \mathrm{Cl})$ & $P$-value & HR $(95 \% \mathrm{CI})$ & $P$-value \\
\hline Age, $\geq 60$ vs. $<60$ years & $0.73(0.56-0.96)$ & 0.023 & $0.76(0.57-1.03)$ & 0.074 \\
\hline Sex, male vs. female & $1.70(1.18-2.47)$ & 0.005 & $1.60(1.08-2.38)$ & 0.020 \\
\hline Child-Pugh class, B-C vs. A & $1.88(1.45-2.43)$ & $<0.001$ & $0.88(0.60-1.29)$ & 0.503 \\
\hline Total bilirubin, $\geq 3$ vs. $<3$ mg/dL & $2.07(1.58-2.71)$ & $<0.001$ & $1.47(1.09-1.97)$ & 0.011 \\
\hline BCLC stage, C-D vs. A-B & $2.01(1.48-2.73)$ & $<0.001$ & $1.60(1.15-2.23)$ & 0.006 \\
\hline Level of bile duct involvement & $1.55(1.19-2.02)$ & 0.001 & $1.23(0.92-1.64)$ & 0.160 \\
\hline \multicolumn{5}{|l|}{ Common or both vs. unilateral } \\
\hline PVTT, present vs. absent & $1.88(1.42-2.48)$ & $<0.001$ & $1.61(1.20-2.17)$ & 0.002 \\
\hline Metastasis, present vs. absent ${ }^{*}$ & $1.86(1.36-2.55)$ & $<0.001$ & $1.56(1.12-2.16)$ & 0.008 \\
\hline \multicolumn{5}{|l|}{ Treatment modality } \\
\hline Resection, present vs. absent & $0.47(0.31-0.72)$ & $<0.001$ & $0.47(0.29-0.74)$ & 0.001 \\
\hline TACE, present vs. absent & $0.46(0.35-0.59)$ & $<0.001$ & $0.39(0.29-0.51)$ & $<0.001$ \\
\hline CTx, present vs. absent & $0.80(0.53-1.19)$ & 0.270 & & \\
\hline
\end{tabular}

HCC, hepatocellular carcinoma; HR, hazard ratio; Cl, confidence interval; BCLC, Barcelona Clinic Liver Cancer; PVTT, portal vein tumor thrombus; TACE, transarterial chemoembolization; CTx, systemic chemotherapy.

*Extra-hepatic metastasis including lung, lymph node, bone or adrenal metastasis. 
prognostic variables affecting the survival of $\mathrm{HCC}$ patients with bile duct invasion, the following host and tumor factors and treatment modalities were statistically significant $(P<0.05)$ : age, male sex, Child-Pugh classification, presence of jaundice, BCLC stage, presence of gross portal vein invasion and extra-hepatic metastasis, level of bile duct involvement, surgical resection, and repeated TACE. In a multivariate analysis using these variables, the effects of male sex, presence of jaundice or portal vein invasion, extrahepatic metastasis, surgical resection and repeated TACE, remained statistically significant. The hazard ratios of surgical resection and repeated TACE were $0.47(P=0.001)$ and $0.39(P<0.001)$, respectively.

\section{DISCUSSION}

The optimal treatment strategy and the prognosis of HCC patients with bile duct invasion have not been established. In this study we examined the survival rates of patients who had HCC complicated with bile duct invasion according to BCLC stage and treatment modality. The majority of patients (74.9\%) were diagnosed with advanced HCC -more than BCLC C stage- and portal vein invasion affected about two-thirds (67.2\%) of the patients. Generally, the prognosis of our patients was dismal, and median survival was only 4.1 months. However, we found that aggressive treatment strategy including biliary drainage, surgical resection, or TACE achieved fairly good survival benefits in some patients.

HCC patients with bile duct invasion are thought to have worse survival than those without bile duct invasion. ${ }^{8-10,26}$ One of the most important reasons for this is that HCC with bile duct invasion is frequently accompanied by portal vein invasion, which is a most potent negative prognostic factor for HCC, reportedly as high as $88 \%$. ${ }^{4,27,28}$ However, when we excluded patients with portal vein invasion, we still found that the survival of $\mathrm{HCC}$ patients with bile duct invasion was poor. Even in early HCC such as BCLC stages A or B, the medial survival of patients with bile duct invasion was markedly shorter than that of those without bile duct invasion described in the prior study (11.6 vs. 32.5 months). ${ }^{29}$ When all the results are combined, bile duct invasion itself seems to be a prognostic factor for poor survival in HCC patients.

Interestingly, of the various HCC staging system, only the Liver Cancer Study Group of Japan (LCSGJ) staging system recognizes that bile duct invasion has as much impact on patients' survival as vascular invasion. ${ }^{2}$ However, the LCSGJ staging system has limitations in that it was based on an analysis of surgical patients.
The results of our study, which included all consecutive patients with HCC and bile duct invasion treated with a variety of modalities, might be useful for improving the staging system of HCC.

Even though the prognosis of HCC patients with bile duct invasion was quite disappointing, we showed that these patients can benefit from aggressive treatment of the HCC and/or a sequential approach, if available. With respect to BCLC stage, patients with stage $\mathrm{C}$ were not recommended for conventional HCC treatments but only for receiving novel agents. However, a previous study showed that patients with non-early stage HCC receiving potentially curative treatment had improved outcomes. ${ }^{30}$ Our results also suggest that there was a modest improvement in survival even in patients with BCLC stage $C$ and bile duct invasion, when treatments were provided.

The long-term outcomes after surgical treatment for HCC with bile duct invasion are still controversial. Some previous studies reported poorer surgical outcomes in these patients than in those without bile duct invasion. 13,15,31,32 However, others have reported that some patients with obstructive jaundice can be treated by hepatic resection, with favorable long-term results. ${ }^{7,10,14,26,33}$ Our data show that surgical resection is associated with better survival are consistent with the latter reports. Even when HCC tumor thrombosis is recognized in the major branches of the bile duct, surgical procedure may be a one of the therapeutic modalities that deserves consideration if there is an expectation of curative and safe surgery. Future prospective studies are needed to establish precise indication of surgical resection in these patients. If HCC recurred after resection, established treatments such as percutaneous ethanol injection, TACE, and RT were effective in our patients.

Unfortunately, the likelihood of undergoing curative resection seems to be low in these patients. ${ }^{31}$ In our case, surgical resection was chosen as initial treatment in only $10.9 \%$ of the patients. Most patients were regarded as inoperable because of advanced disease and poor hepatic functional reserve, or the presence of portal vein tumor thrombus. In general, TACE has not been actively recommended in patients with bile duct invasion. ${ }^{34}$ Nonetheless, we found that those patients who received TACE had better survival outcomes than those receiving conservative management, or systemic chemotherapy, especially in the case of BCLC stage C patients. Further study is needed to assess the safety of TACE in such patients.

Patients who responded well to bile duct drainage had significantly improved survival. In accord with our results, several previous reports have suggested that endoscopic biliary drainage is 
one of the most effective treatments for patients with malignant biliary stenosis. ${ }^{11,21-24}$ We recommend that early effective bile duct drainage should be the first step in non-surgical treatment, since it can improve hepatic function and create the conditions for subsequent treatment of the primary disease.

Certain limitations should be taken into consideration when interpreting the results of the current study. The first identified also in our results is the retrospective nature of the work, which could lead to selection bias in determining the choice of treatment regimen. Based on our results, the prospective randomized study regarding beneficial treatment options in these patients is warranted. Second, we could not directly compare our results with those of patients without bile duct invasion. Therefore we could not obtain unambiguous evidence for the prognostic significance of bile duct invasion in HCC patients. However, the outcomes of this, the largest study of consecutive patients regardless of HCC stage or treatment, permit us to suggest a possible role of aggressive conventional treatment in HCC patients with bile duct invasion. Third, the presence of bile duct invasion was determined by CT imaging without pathologic confirmation in some of the included patients. Because the patients were diagnosed as HCC according to AASLD and EASL criteria, most of them did not undergo a pathologic examination. However, in relation to usual clinical practice in the diagnosis and treatment of $\mathrm{HCC}$, using $\mathrm{CT}$ images for evaluating the presence of bile duct invasion may be quite appropriate as it reflects the real clinical situation.

In conclusion, the survival of HCC patients with bile duct invasion at initial diagnosis is generally poor. However, aggressive treatment for HCC could provide survival benefits in those patients with preserved liver function, especially in those with $B C L C$ stage C. Our survival data could be used as historical controls in future clinical trials evaluating treatments in $\mathrm{HCC}$ patients with bile duct invasion.

\section{Conflicts of Interest}

The authors have no conflicts to disclose.

\section{REFERENCES}

1. Ferlay J, Shin HR, Bray F, Forman D, Mathers C, Parkin DM. Estimates of worldwide burden of cancer in 2008: GLOBOCAN 2008. Int J Cancer 2010;127:2893-2917.

2. Minagawa M, Ikai I, Matsuyama Y, Yamaoka Y, Makuuchi M. Staging of hepatocellular carcinoma: assessment of the Japanese TNM and AJCC/UICC TNM systems in a cohort of 13,772 patients in Ja- pan. Ann Surg 2007;245:909-922.

3. Llovet JM, Brú C, Bruix J. Prognosis of hepatocellular carcinoma: the BCLC staging classification. Semin Liver Dis 1999;19:329-338.

4. Kojiro M, Kawabata K, Kawano Y, Shirai F, Takemoto N, Nakashima T. Hepatocellular carcinoma presenting as intrabile duct tumor growth: a clinicopathologic study of 24 cases. Cancer 1982;49:2144-2147.

5. Chen MF, Jan YY, Jeng LB, Hwang TL, Wang CS, Chen SC. Obstructive jaundice secondary to ruptured hepatocellular carcinoma into the common bile duct. Surgical experiences of 20 cases. Cancer 1994;73:1335-1340.

6. Peng BG, Liang LJ, Li SQ, Zhou F, Hua YP, Luo SM. Surgical treatment of hepatocellular carcinoma with bile duct tumor thrombi. World J Gastroenterol 2005;11:3966-3969.

7. Lau WY, Leung JW, Li AK. Management of hepatocellular carcinoma presenting as obstructive jaundice. Am J Surg 1990;160:280-282.

8. Huang JF, Wang LY, Lin ZY, Chen SC, Hsieh MY, Chuang WL, et al. Incidence and clinical outcome of icteric type hepatocellular carcinoma. J Gastroenterol Hepatol 2002;17:190-195.

9. Suh YG, Kim DY, Han KH, Seong J. Effective biliary drainage and proper treatment improve outcomes of hepatocellular carcinoma with obstructive jaundice. Gut Liver 2014;8:526-535.

10. Huang GT, Sheu JC, Lee HS, Lai MY, Wang TH, Chen DS. Icteric type hepatocellular carcinoma: revisited 20 years later. J Gastroenterol 1998:33:53-56.

11. Choi J, Shim JH, Park DH, Lee SS, Seo DW, Lee SK, et al. Clinical usefulness of endoscopic palliation in patients with biliary obstruction caused by hepatocellular carcinoma. Digestion 2013;88:87-94.

12. Kasai Y, Hatano E, Seo S, Taura K, Yasuchika K, Uemoto S. Hepatocellular carcinoma with bile duct tumor thrombus: surgical outcomes and the prognostic impact of concomitant major vascular invasion. World J Surg 2015;39:1485-1493.

13. Meng KW, Dong M, Zhang WG, Huang QX. Clinical characteristics and surgical prognosis of hepatocellular carcinoma with bile duct invasion. Gastroenterol Res Pract 2014;2014:604971.

14. Moon DB, Hwang S, Wang HJ, Yun SS, Kim KS, Lee YJ, et al. Surgical outcomes of hepatocellular carcinoma with bile duct tumor thrombus: a Korean multicenter study. World J Surg 2013;37:443-451.

15. Yu XH, Xu LB, Liu C, Zhang R, Wang J. Clinicopathological characteristics of 20 cases of hepatocellular carcinoma with bile duct tumor thrombi. Dig Dis Sci 2011;56:252-259.

16. Edge SB, Compton CC. The American Joint Committee on Cancer: the 7th edition of the AJCC cancer staging manual and the future of TNM. Ann Surg Oncol 2010;17:1471-1474.

17. Chopra S, Chintapalli KN, Ramakrishna K, Rhim H, Dodd GD 3rd. Helical CT cholangiography with oral cholecystographic contrast material. Radiology 2000;214:596-601.

18. Holm AN, Gerke H. What should be done with a dilated bile duct? Curr Gastroenterol Rep 2010;12:150-156. 
19. European Association For The Study Of The Liver; European Organisation For Research And Treatment Of Cancer. EASL-EORTC clinical practice guidelines: management of hepatocellular carcinoma. J Hepatol 2012;56:908-943.

20. Bruix J, Sherman M; American Association for the Study of Liver Diseases. Management of hepatocellular carcinoma: an update. Hepatology 2011;53:1020-1022.

21. Paik WH, Park YS, Hwang JH, Lee SH, Yoon CJ, Kang SG, et al. Palliative treatment with self-expandable metallic stents in patients with advanced type III or IV hilar cholangiocarcinoma: a percutaneous versus endoscopic approach. Gastrointest Endosc 2009;69:55-62.

22. Yoon WJ, Lee JK, Lee KH, Lee WJ, Ryu JK, Kim YT, et al. A comparison of covered and uncovered wallstents for the management of distal malignant biliary obstruction. Gastrointest Endosc 2006;63:9961000.

23. De Palma GD, Galloro G, Siciliano S, Iovino P, Catanzano C. Unilateral versus bilateral endoscopic hepatic duct drainage in patients with malignant hilar biliary obstruction: results of a prospective, randomized, and controlled study. Gastrointest Endosc 2001;53:547-553.

24. Hong HP, Kim SK, Seo TS. Percutaneous metallic stents in patients with obstructive jaundice due to hepatocellular carcinoma. J Vasc Interv Radiol 2008;19:748-754.

25. Kim KM, Kim JH, Park IS, Ko GY, Yoon HK, Sung KB, et al. Reappraisal of repeated transarterial chemoembolization in the treatment of hepatocellular carcinoma with portal vein invasion. J Gastroenterol Hepatol 2009;24:806-814.

26. Lau WY, Leung KL, Leung TW, Ho S, Chan M, Liew CK, et al. Obstructive jaundice secondary to hepatocellular carcinoma. Surg Oncol 1995;4:303-308
27. Satoh S, Ikai I, Honda G, Okabe H, Takeyama O, Yamamoto Y, et al. Clinicopathologic evaluation of hepatocellular carcinoma with bile duct thrombi. Surgery 2000;128:779-783.

28. Shiomi M, Kamiya J, Nagino M, Uesaka K, Sano T, Hayakawa N, et al. Hepatocellular carcinoma with biliary tumor thrombi: aggressive operative approach after appropriate preoperative management. Surgery 2001;129:692-698.

29. Kwak HW, Park JW, Nam BH, Yu A, Woo SM, Kim TH, et al. Clinical outcomes of a cohort series of patients with hepatocellular carcinoma in a hepatitis B virus-endemic area. J Gastroenterol Hepatol 2014;29:820-829.

30. Kanwal F, Befeler A, Chari RS, Marrero J, Kahn J, Afdhal N, et al. Potentially curative treatment in patients with hepatocellular cancer-results from the liver cancer research network. Aliment Pharmacol Ther 2012;36:257-265.

31. Shao W, Sui C, Liu Z, Yang J, Zhou Y. Surgical outcome of hepatocellular carcinoma patients with biliary tumor thrombi. World J Surg Oncol 2011;9:2.

32. Ikenaga N, Chijiiwa K, Otani K, Ohuchida J, Uchiyama S, Kondo K. Clinicopathologic characteristics of hepatocellular carcinoma with bile duct invasion. J Gastrointest Surg 2009;13:492-497.

33. Hu J, Pi Z, Yu MY, Li Y, Xiong S. Obstructive jaundice caused by tumor emboli from hepatocellular carcinoma. Am Surg 1999;65:406-410.

34. Raoul JL, Sangro B, Forner A, Mazzaferro V, Piscaglia F, Bolondi L, et al. Evolving strategies for the management of intermediate-stage hepatocellular carcinoma: available evidence and expert opinion on the use of transarterial chemoembolization. Cancer Treat Rev 2011;37:212-220. 